\title{
Transarterial embolization for the treatment of complicated liver hemangiomas: A report of two cases and review of the literature
}

\author{
Maria Kalogirou', Danai Chourmouzi ${ }^{2}$, loannis Dedes ${ }^{2}$, Stefania Kiapidou', Evangelos Akriviadis ${ }^{1}$, and \\ Emmanouil Sinakos ${ }^{1}$ \\ $14^{\text {th }}$ Department of Internal Medicine, Aristotle University of Thessaloniki, Hippokration General Hospital, Thessaloniki; ${ }^{2}$ European \\ Interbalkan Medical Center, Thessaloniki, Greece
}

Dear Editor,

Hemangioma is the most common benign liver tumor with a frequency of $0.4-7.3 \%$ in autopsy series. It is the second most common tumor seen in the liver after metastases. These lesions are mostly asymptomatic and incidentally diagnosed. However, they can become symptomatic with signs and symptoms varying from abdominal fullness or pain, fatigue, jaundice, disseminated intravascular coagulation, to life-threatening spontaneous rupture. ${ }^{2}$ Giant liver hemangiomas are defined by a diameter larger than 5 $\mathrm{cm}{ }^{3,4}$ In asymptomatic patients with a giant liver hemangioma, observation is an accepted policy. ${ }^{3,4}$ Symptomatic liver hemangiomas are, generally, treated by surgery (hepatic resection or enucleation, open, laparoscopic or robotic). However, surgery of giant liver hemangiomas can cause massive intraoperative hemorrhage, with high mortality rate. 1 Furthermore, many patients with spontaneous rupture of hepatic hemangiomas, are too weak to tolerate surgery, due to hypovolemia. ${ }^{4}$ Over the past years, transarterial embolization (TAE) has gained ground as an alternative therapeutic strategy for symptomatic hemangiomas, mostly preoperatively, but also as a definite therapy. TAE can achieve tumor shrinkage and symptoms remission, with few complications, being indicated especially in patients with high surgical risk.

We herein describe 2 cases of bleeding giant liver hemangiomas that have been successfully treated, solely with TAE.

In the first case, a 43-year-old male with a history of a giant hepatic hemangioma presented with acute right upper quadrant abdominal pain of 3 days' duration with no other related symptoms. The giant hemangioma had been diagnosed about 4 years ago, when the patient was investigated for persistent mild abdominal discomfort. At that time, the lesion size was $6 \mathrm{~cm}$ and it kept growing to reach a final size of $7.5 \mathrm{~cm}$.

Physical examination revealed only mild abdominal tenderness in the right upper quadrant. Of note, vital signs were within normal limits. The results of laboratory investigations included a normal complete blood count (hemoglobin [Hb] $14.6 \mathrm{~g} / \mathrm{dL}$, hematocrit [Ht] 42.9\%, platelet counts [plt] 228,000/ $\mathrm{L}$ ) and elevated liver enzymes (aspartate transaminase [AST], $100 \mathrm{U} / \mathrm{L}$, alanine transaminase [ALT] $123 \mathrm{U} / \mathrm{L}$ ). A dynamic computed tomography (CT) showed the presence of an exophytic hemangioma in segments $\mathrm{VI}$ and $\mathrm{VII}$, with signs of rupture and hemorrhage, as well as an asso-

\section{Abbreviations:}

$\mathrm{CT}$, computed tomography; MRI, magnetic resonance imaging; RF, radiofrequency; TACE, transarterial chemoembolization; TAE, transarterial embolization

\section{Corresponding author : Maria Kalogirou}

$4^{\text {th }}$ Department of Internal Medicine, Aristotle University of Thessaloniki, Hippokratio General Hospital, Thessaloniki, Konstantinoupoleos Street 49, 54642, Greece

Tel: +30 6989091601

E-mail:maria.kalogi32@gmail.com

https://orcid.org/0000-0001-9985-4038

Received : Feb. 25, 2018/ Accepted : Feb. 26, 2018

Copyright ( $\odot 2018$ by Korean Association for the Study of the Live

This is an Open Access article distributed under the terms of the Creative Commons Attribution Non-Commercial License (http://creativecommons.org/licenses/by-nc/3.0/) which permits unrestricted non-commercial use, distribution, and reproduction in any medium, provided the original work is properly cited. 
ciated subhepatic fluid collection (Fig. 1A-E). Small cystic lesions were also observed.

The patient underwent superselective embolization (Fig. 1F, G). Six $\mathrm{mL}$ of lipiodol were administered. The embolic agent used was microspheres, with a size ranging between 100 and 500 microns. After TAE procedure, he developed mild post-TAE syndrome that resolved spontaneously. A follow-up CT scan was performed one month after TAE and demonstrated reduction in the size of the hemangioma from $7.5 \mathrm{~cm}$ before TAE to $6.9 \mathrm{~cm}$ after TAE. (Fig. 1H). The patient was asymptomatic during follow-up of 10 months after the TAE.

The second case, a 79-year-old female was admitted to our department with the diagnosis of a giant liver hemangioma. The patient had experienced severe fatigue and anorexia over the past month. Her past medical history was significant for adrenalectomy, 45 years ago, and long-term hydrocortisone treatment. Crosssectional imaging with $\mathrm{CT}$ revealed an exophytic mass located in the right lobe of the liver. This lesion had significantly increased in size as compared with the previous $\mathrm{CT}$ examination 2 years ago (from 7.7 to $15 \mathrm{~cm}$ ) (Fig. 2A).

Clinical examination showed normal vital signs, tenderness in the epigastrium and right upper quadrant, as well as severe peripheral edema. The laboratory results were: $\mathrm{Hb} 11.20 \mathrm{~g} / \mathrm{dL}, \mathrm{Ht}$

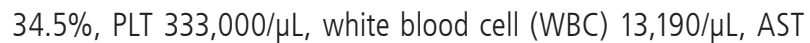
17 U/L, ALT 10 U/L, alkaline phosphatase (ALP) 167 U/L, $\gamma$-GT 156 U/L, Alb $2.5 \mathrm{~g} / \mathrm{dL}$ and CRP $19.4 \mathrm{mg} / \mathrm{dL}$ (reference range of CRP: 0-0.5 mg/dL). Subsequently, an magnetic resonance imaging (MRI) of the abdomen was performed, which exhibited progressive nodular enhancement of the lesion, with signs of central necrosis or thrombotic degeneration (Fig. 2B, C). Other smaller liver hemangiomas were also detected.

During hospitalization, the patient developed non-cardiogenic pulmonary edema. Cardiac evaluation appeared normal and the occurrence of pulmonary edema was thought to be associated with the hyperdynamic circulation caused by the hemangioma. Laboratory tests, 4 days after admission, although not fully compatible, were suggestive of a Kasabach-Merritt syndrome (9.50 $\mathrm{g} / \mathrm{dL}$, Ht 29.4\%, PLT 110,000/ $\mathrm{L}$, the international normalized ratio for prothrombin time [INR] 1.57), combined with cholestasis (ALP177 U/L, $\gamma$-GT $166 \mathrm{U} / \mathrm{L}$ ), elevated CRP levels $(23.4 \mathrm{mg} / \mathrm{dL}$ ), leukocytosis (WBC 29,690/ $\mathrm{LL}$ ) and mild elevation of liver transaminases (AST $86 \mathrm{U} / \mathrm{L}$, ALT $34 \mathrm{U} / \mathrm{L}$ ).

The patient was initially treated with intravenous fluids, antibiotics, diuretics and steroids for five days. On the sixth day of hospitalization she underwent a successful selective TAE (Fig. 2D, E). Eight $\mathrm{mL}$ of lipiodol were administered. The embolic agent used
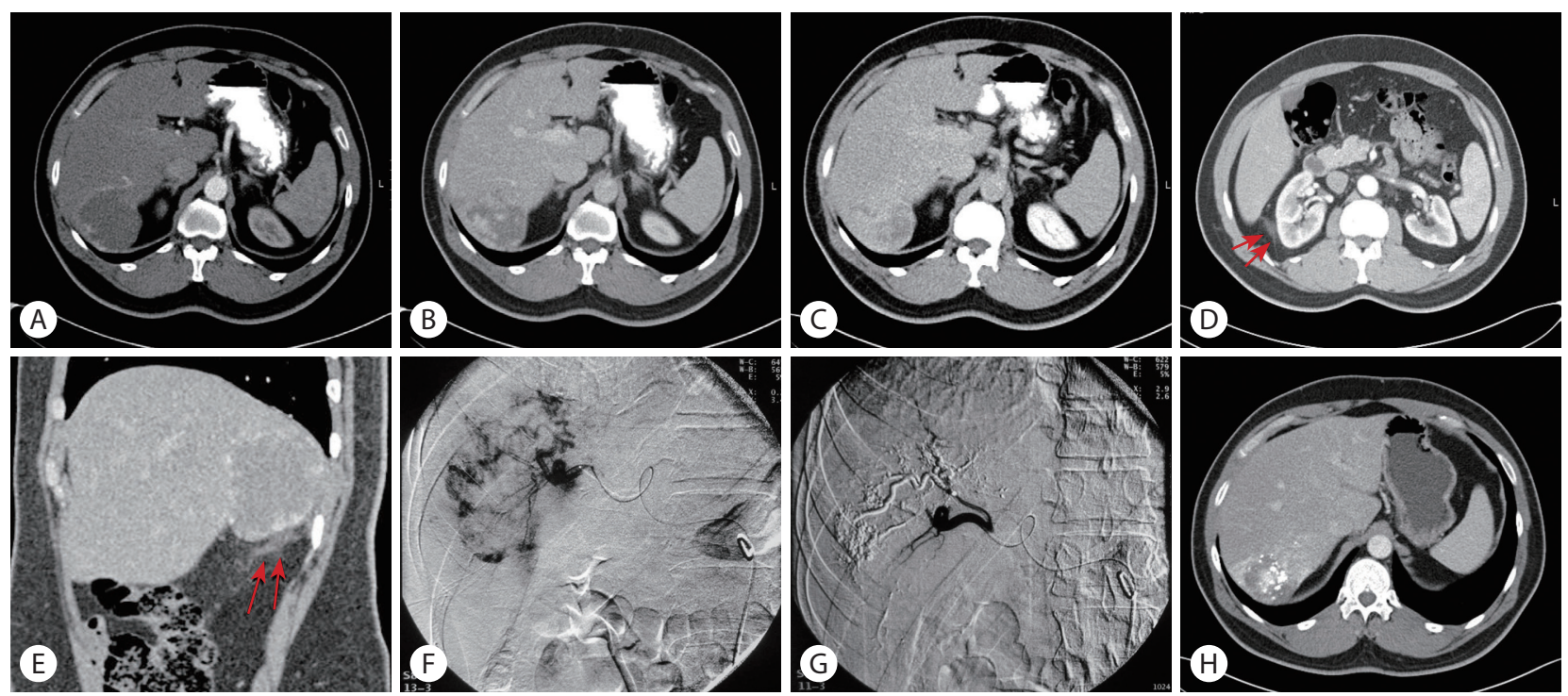

Figure 1. (A) Axial post-contrast CT (arterial phase) showed an exophytic hypodense lesion arising from the right lobe of the liver with peripheral rim enhancement. (B) In venous phase, the lesion showed peripheral discontinuous enhancing nodules. (C) In delayed phase, persistent incomplete filling was seen. Axial CT scan in a lower level (D) and sagittal reconstruction (E) showed sub-hepatic linear high attenuation-fat stranding, a sign compatible with small hemorrhage (arrows, D, E). (F) Arterial phase of right hepatic angiogram showed absence of neovascularity and characteristic fluffy staining of contrast in the liver hemangioma. (G) Selective hepatic angiogram obtained after embolization showed a complete shutdown of the vascularity, pruning of the peripheral vessels and lipiodol accumulation. (H) Post-embolization CT showed lipiodol accumulation and significant decrease of volume of the lesion. 

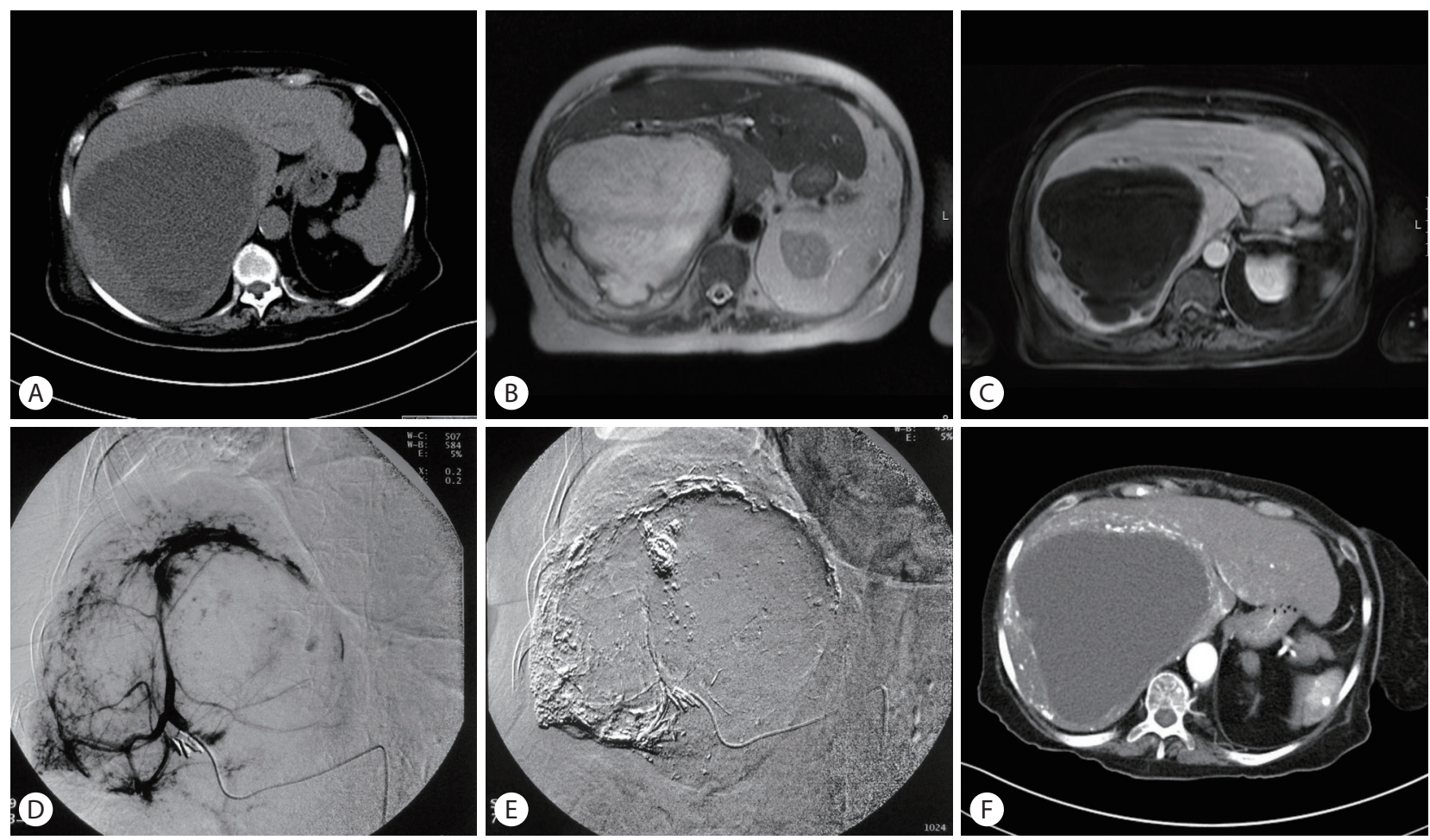

Figure 2. (A) Axial pre-contrast CT scan showed a large hypodense lesion within the right liver lobe. (B) Axial T2-weighted images showed a large high signal intensity mass. (C) On post contrast vibe image the mass showed peripheral nodular enhancement. Most of lesion was not enhanced. (D) Arterial phase of hepatic angiogram showed characteristic pooling of contrast in the liver hemangioma. (E) Post TAE angiogram showed lipiodol accumulation. (F) Post embolization CT scan showed peripheral lipiodol accumulation.

was microspheres, with a size ranging from 100 to 700 microns. The patient experienced no TAE-related complications. After the procedure, laboratory values returned to normal and symptoms gradually resolved. At follow-up of 9 months after TAE, symptoms had resolved and a CT scan showed a slight decrease in size of the tumor (Fig. 2F).

The management of liver hemangiomas generally consists of counseling and periodic follow-up. Treatment is reserved for special cases and this should be decided based on the size and location of the tumor. ${ }^{5}$ Surgical enucleation has been, traditionally, the treatment of choice. ${ }^{3}$ Absolute indications for surgery are spontaneous or traumatic rupture with hemoperitoneum, intratumoral bleeding and consumptive coagulopathy (Kasabach-Merritt Syndrome). ${ }^{5}$ In the recent years, TAE has been increasingly used as a preoperative treatment method for bleeding giant hemangiomas in order to achieve hemodynamic stability of the patient, before definite surgical treatment. ${ }^{2}$ However, TAE alone could be used as a treatment method for bleeding hemangiomas without follow-up surgical intervention. We herein describe two cases of bleeding hemangiomas being successfully treated solely with TAE.

The first patient experienced a spontaneous rupture of the hemangioma. In the second case, in addition to hemorrhage, significant systemic derangement occurred leading to hyperdynamic circulation and non-cardiac pulmonary edema. TAE was uneventful in both patients. Symptoms gradually resolved and follow-up imaging showed size reduction in both cases.

The successful use of TAE before surgery of a ruptured hemangioma was first reported by Yamamoto, et al in 1991. ${ }^{5}$ According to Srinivasa, et al. TAE is a successful hemostatic method in almost $80 \%$ of cases. ${ }^{6}$ Lipiodol is a microvessel embolic agent that has tumor-philic properties, and can block the tumor's blood flow. When injected into the hepatic artery, it selectively concentrates within tumor nodules, where it is trapped for several weeks to over 12 months, due to a siphoning effect from hypervascularization of the tumor vessels and an absence of Kupffer cells inside tumor tissues. ${ }^{8}$ There is no consensus regarding the embolic agents used in TAE. In the present study, both patients were embolized using lipiodol and microspheres, as this combination appeared to have the best overall results in our center. It is widely believed that microspheres have an additive effect to lipiodol.

Previous reports have showed favorable outcomes of TAE as a sole treatment method for liver hemangiomas. Zeng, et al. 
managed 98 cases with hemangioma only with TAE. Only 2 of them eventually required a surgical intervention. ${ }^{9}$ Firouznia, et al. performed TAE on 20 patients with liver hemangioma as a sole therapy. None of these patients required another intervention, including surgery, using a follow-up period of 6 months after TAE. ${ }^{10}$ In 2015, Sun, et al. reported 26 patients with hemangioma treated with TAE. Follow-up with clinical and imaging examinations showed resolution of symptoms and decrease in size of lesions in all patients. ${ }^{7}$ According to Toro, et al., surgery of giant hemangiomas of the liver either with resection or with enucleation can cause significant, difficult to control intraoperative hemorrhage with high mortality risk for the patients.' In these cases, TAE could be a useful alternative. In the same review, the authors reported that TAE had a mortality rate of $0 \%$ and morbidity rate of $54 \%$ as compared to $0-3 \%$ and $10-27 \%$ of surgery, respectively. Both methods had recurrence rate of $0 \%$.

However, TAE is still controversial due to the risk of ectopic embolization and ischemia, infection, intrahepatic bile duct injury and liver dysfunction. ${ }^{5,7}$ Zeng, et al. reported only transient liver dysfunction in 69 patients, the vast majority of whom returned to normal within 2 weeks, while Sun, et al. reported no bile duct injury and clinically significant elevated liver enzymes in only one patient. ${ }^{7.9} \mathrm{~A}$ major limitation of our study was the relatively short follow-up time (overall 10 months for the first patient and 9 for the second). Furthermore, most of the reports published refer to TAE as a preoperative and not a definite treatment modality. Therefore, more studies with long-term follow-up should be performed to establish the effectiveness and safety of TAE as a sole therapy for symptomatic hemangiomas.

Recently, transarterial chemoembolization (TACE) has been proposed as an alternative therapeutic strategy for the treatment of giant liver hemangiomas. In 2015, Li, et al. performed selective TACE using pingyangmycin-lipiodol on 836 patients with symptomatic giant liver hemangiomas and the patients were followedup from 1 year to 10 years. The success rate of the procedure was $100 \%$. Symptom alleviation was achieved on all patients, while a significant decrease in the mean diameter of the hemangiomas was reported (from $9.6+/-0.8 \mathrm{~cm}$ to $3.6+/-0.5 \mathrm{~cm}$ ). ${ }^{11}$ In 2016, Ji, et al. treated 15 patients with giant liver hemangiomas with TAE, using a pingyangmycin-lipiodol mixture, combined with radiofrequency (RF) ablation one month after TAE. ${ }^{12}$ The mean diameter of the hemangiomas was significantly reduced 6 months after the ablation (from $13.3 \pm 2.2 \mathrm{~cm}$ to $4.9 \pm 1.6 \mathrm{~cm}$ ). Four patients experienced minor ablation-related complications. The researchers suggested that RF ablation combined with TAE is associated with lower incidence of ablation-related complications due to shorter ablative times. ${ }^{12}$ Although this innovative approach seems to be very promising in the management of giant liver hemangiomas, future randomized controlled trials are necessary to compare the efficacy between TAE and TACE in those patients.

In conclusion, TAE is an effective minimal invasive treatment modality for complicated liver hemangiomas. This treatment approach is ideal for patients with high surgical risk due to its low complication rate. TAE could be used as a primary treatment for giant hemangiomas, standing as a valid alternative option to surgery in selected cases.

\section{Authors contribution:}

Kalogirou M wrote the manuscript; Chourmouzi D performed radiological diagnosis; Dedes I performed transarterial embolization; Kiapidou S collected patients' data; Akriviadis E assessed the patients; Sinakos E critically reviewed the manuscript.

\section{Conflicts of Interest}

The authors have no conflicts to disclose.

\section{REFERENCES}

1. Toro A, Mahfouz AE, Ardiri A, Malaguarnera M, Malaguarnera G, Loria $F$, et al. What is changing in indications and treatment of hepatic hemangiomas. A review. Ann Hepatol 2014;13:327-339.

2. Donati M, Stavrou GA, Donati A, Oldhafer KJ. The risk of spontaneous rupture of liver hemangiomas: a critical review of the literature. $J$ Hepatobiliary Pancreat Sci 2011;18:797-805.

3. Hoekstra LT, Bieze M, Erdogan D, Roelofs JJ, Beuers UH, van Gulik TM. Management of giant liver hemangiomas: an update. Expert Rev Gastroenterol Hepatol 2013;7:263-268.

4. Jangjoo A, Mehrabi Bahar M, Aliakbarian M. Ruptured giant hepatic hemangioma: report of a case. Acta Medica Iranica 2010;48:419422.

5. Ribeiro MA Jr, Papaiordanou F, Gonçalves JM, Chaib E. Spontaneous rupture of hepatic hemangiomas: a review of the literature. World J Hepatol 2010;2:428-433.

6. Srinivasa S, Lee WG, Aldameh A, Koea JB. Spontaneous hepatic haemorrhage: a review of pathogenesis, aetiology and treatment. HPB (Oxford) 2015;17:872-880.

7. Sun JH, Nie CH, Zhang YL, Zhou GH, Ai J, Zhou TY, et al. Transcatheter arterial embolization alone for giant hepatic hemangioma. PLoS One 2015;10:e0135158.

8. Shin SW. The current practice of transarterial chemoembolization 
Maria Kalogirou, et al. Transarterial embolization in complicated liver hemangiomas

for the treatment of hepatocellular carcinoma. Korean J Radiol 2009;10:425-434.

9. Zeng Q, Li Y, Chen Y, Ouyang Y, He X, Zhang H. Gigantic cavernous hemangioma of the liver treated by intra-arterial embolization with pingyangmycin-lipiodol emulsion: a multi-center study. Cardiovasc Intervent Radiol 2004;27:481-485.

10. Firouznia K, Ghanaati H, Alavian SM, Nassiri Toosi M, Ebrahimi Daryani $\mathrm{N}$, Jalali $\mathrm{AH}$, et al. Management of liver hemangioma using trans-catheter arterial embolization. Hepat Mon 2014;14:e25788.

11. Li Y, Jia Y, Li S, Wang W, Wang Z, Wang Y, et al. Transarterial chemoembolization of giant liver haemangioma: a multi-center study with 836 cases. Cell Biochem Biophys 2015;73:469-472.

12. Ji J, Gao J, Zhao L, Tu J, Song J, Sun W. Computed tomography-guided radiofrequency ablation following transcatheter arterial embolization in treatment of large hepatic hemangiomas. Medicine (Baltimore) 2016;95:e3402. 